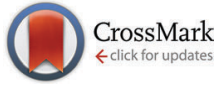

Cite this: J. Mater. Chem. C, 2017, 5, 3296

\title{
Nanoscale interfacial electroactivity in PVDF/PVDF-TrFE blended films with enhanced dielectric and ferroelectric properties $\dagger$
}

\author{
Nan Meng, Xiaojing Zhu, Rui Mao, Michael John Reece and Emiliano Bilotti*
}

\begin{abstract}
The typical limitations of ferroelectric polymers like poly(vinylidene fluoride) (PVDF) - low crystallinity and indirect ferroelectric $\beta$-phase crystallization - and poly(vinylidene fluoride-trifluoroethylene) (PVDF-TrFE) - high materials and processing costs and a low Curie point - are tackled by a simple and industrially viable melt blending approach. Despite the immiscible nature of PVDF and PVDF-TrFE, strong interactions exist between the two polymers, which substantially affect the morphology and texture of the blends as well as their dielectric and ferroelectric properties. Surprisingly, minor amounts of PVDF-TrFE lead to a significant increase in the $\beta$-phase content and preferred orientation of PVDF, well beyond the rule-of-mixtures. Moreover, the blends exhibit maximum increases in the dielectric constant of $80 \%$ and $30 \%$, respectively, compared with pure PVDF and PVDF-TrFE. The ferroelectric remnant polarization increases from 0.040 to $0.077 \mathrm{C} \mathrm{m}^{-2}$, while the coercive field decreases from 75 to $32 \mathrm{kV} \mathrm{mm}^{-1}$ with increasing PVDF-TrFE content from 0 to $40 \mathrm{wt} \%$. The enhancement of properties is explained by the strong interactions at the interfaces between PVDF and PVDF-TrFE, which also suppress the Curie transition of PVDF-TrFE, providing a potentially increased working temperature range for blended films, which is important in applications like non-volatile energy storage devices, ferroelectric field-effect transistors and touch sensors.
\end{abstract}

Received 11th January 2017, Accepted 28th February 2017 DOI: $10.1039 / \mathrm{c} 7 \mathrm{tc0} 0162 \mathrm{~b}$

rsc.li/materials-c

\section{Introduction}

Polymer-based ferroelectric materials have low processing temperatures, high electrical resistivity and excellent flexibility compared to ceramic materials, which make them of interest for flexible electronic devices, such as memory devices and sensors. ${ }^{1-6}$ Poly(vinylidene fluoride) (PVDF) and its copolymers with trifluoroethylene (PVDF-TrFE) can be easily fabricated into films with good ferroelectric properties. Unlike ferroelectric ceramics, whose polar properties originate from ion displacement inside the crystal unit cell, the polar properties of ferroelectric polymers are due to the polar groups in the crystalline polymer structure. As a consequence of this, the coercive field of ferroelectric polymers is high $\left(>50 \mathrm{kV} \mathrm{mm}^{-1}\right) .^{7}$

PVDF, a semi-crystalline polymer, shows at least four polymorphs $\left(\alpha-, \beta-, \gamma\right.$ - and $\delta$-). ${ }^{7}$ The crystallization from the melt normally leads to the non-polar $\alpha$-phase, which possesses a trans-gauche chain conformation resulting in the self-cancelation of the dipoles. ${ }^{7}$ The $\alpha$-phase PVDF can be transformed into the $\gamma$-phase through thermal treatments ${ }^{8}$ and into the $\delta$-phase

School of Engineering and Materials Science, Queen Mary University of London, Mile End Road, E1 4NS, UK. E-mail: e.bilotti@qmul.ac.uk

$\dagger$ Electronic supplementary information (ESI) available. See DOI: 10.1039/c7tc00162b through poling under a high electric field $\left(\sim 150 \mathrm{kV} \mathrm{mm}^{-1}\right) \cdot{ }^{9,10}$ While the $\gamma$ - and $\delta$ - phases are polar to some extent, the $\beta$-phase displays the best piezoelectric and ferroelectric properties. The polar direction of $\beta$-PVDF is along its $b$-axis, while the polymer chains are aligned with the $c$-axis. ${ }^{7} \beta$-phase PVDF can be made by mechanically stretching the $\alpha$-phase $\operatorname{PVDF}^{11}$ or poling $\alpha$ - and $\delta$-PVDF under even higher electric fields $\left(>500 \mathrm{kV} \mathrm{mm}^{-1}\right){ }^{10}$

On the other hand, the copolymer PVDF-TrFE with a TrFE content in the range of $20-35 \mathrm{~mol} \%$ easily crystallizes as the ferroelectric $\beta$-phase, independent of the processing routes or post-treatments. ${ }^{12}$ Moreover, the crystallinity of PVDF-TrFE is much higher compared with $\sim 50 \%$ for PVDF, and can reach $90 \%$ after annealing in the temperature range between the Curie and melting points. ${ }^{13}$ This is due to the increased chain mobility of PVDF-TrFE, leading to an increase in the lamella thickness. However, the large scale application of PVDF-TrFE is impeded by its time-consuming and expensive synthesis, ${ }^{12}$ along with a limited working temperature range due to the existence of Curie transition.

Blending is a common strategy to modify the properties of a base polymer by combining the desirable characteristics of different polymers. Miscibility is an important issue related to the evaluation of blends. PVDF blends with amorphous polymers containing a carbonyl group (e.g. polymethyl methacrylate PMMA) exhibit good miscibility in the whole composition range 
due to the contribution from the hydrogen bonding between the double-bonded oxygen of the carbonyl group and the acidic hydrogen of the $\mathrm{CH}_{2}-\mathrm{CF}_{2}$ group. ${ }^{14-16}$ However, the crystallization of PVDF and its spontaneous polarization are suppressed with the addition of amorphous polymers. ${ }^{15,17}$ As a result, in order to maintain or even enhance its ferroelectric properties, blending with fluorine polymers is more likely to be effective. ${ }^{18}$

Tanaka et al. ${ }^{19}$ investigated the miscibility of PVDF/PVDF-TrFE blends. Based on the observation of two distinct fusion peaks in the DSC heating run, PVDF was found to be immiscible with PVDF-TrFE regardless of the composition of the copolymer. Gregorio et $a l^{20}$ came to the same conclusion, but they suggested that PVDF and PVDF-TrFE displayed miscibility on the lamellae level due to the fact that pure PVDF showed axial morphology, while the blends displayed a homogenously distributed irregular texture, suggesting that PVDF-TrFE molecules segregated to the regions between the PVDF spherulites. However, they did not report the electric properties of their blended films. Despite the immiscibility of the polymers, there is evidence of strong interaction in PVDF/PVDF-TrFE blends, which might have a significant effect on the dielectric and ferroelectric properties of the blends.

In this work, PVDF was blended with PVDF-TrFE using melt extrusion, which, to the best of our knowledge, has never been reported before. On the basis of our previous study, ${ }^{21}$ melt extruded PVDF-TrFE films exhibited remarkable ferroelectric properties due to high crystallinity and highly preferred crystalline orientation. It is proposed that the presence of PVDF-TrFE could enhance the crystallization of PVDF into the $\beta$-phase and generate the preferred orientation of its polymer chains.

\section{Experimental}

\section{Materials}

PVDF was purchased from Sigma Aldrich Chemical Co. The average molecular weight of PVDF was about $180 \mathrm{~kg} \mathrm{~mol}^{-1}\left(M_{\mathrm{w}}\right)$ and $71 \mathrm{~kg} \mathrm{~mol}{ }^{-1}\left(M_{\mathrm{n}}\right)$. PVDF-TrFE of composition $77 / 23 \mathrm{~mol} \%$ was purchased from Piezotech S.A.S. (France). The average molecular weight of PVDF-TrFE was $210 \mathrm{~kg} \mathrm{~mol}^{-1}\left(M_{\mathrm{w}}\right)$ and $100 \mathrm{~kg} \mathrm{~mol}{ }^{-1}\left(M_{\mathrm{n}}\right){ }^{22}$

\section{Sample preparation}

PVDF and PVDF-TrFE were melt-blended using a DSM X'plore 15 Mini-extruder (Xplore Instruments, Geleen, The Netherlands) at $205{ }^{\circ} \mathrm{C}$ and $60 \mathrm{rpm}$ for $10 \mathrm{~min}$. The weight ratios of PVDF/PVDF-TrFE were set to 100/0, 90/10, 80/20, 70/30, 60/40 and 0/100. A slit die with a gauge of $200 \mu \mathrm{m}$ was used to produce films. The films were collected by a roller at $180 \mathrm{~mm} \min ^{-1}$ and ambient temperature. The films were then clamped and annealed at $100{ }^{\circ} \mathrm{C}$ for 2 hours. The thickness of the films was about $20 \mu \mathrm{m}$. For electrical measurements, gold was vacuum sputtered on both sides of the films to form electrodes.

\section{Instrumentation}

Fourier transform infrared spectroscopy (FTIR) (Tensor 27, Bruker Optik GmbH, Ettlingen, Germany) was used to characterize the crystalline phases. Five specimens were characterized for every composition of the film. To complement the FTIR data, the crystalline phases were also determined using one-dimensional wide-angle X-ray diffraction (1D-WAXD) patterns which were obtained using a Bragg-Brentano geometry X-ray diffractometer (X'Pert Pro, PANalytical, Almelo, The Netherlands) with $\mathrm{Cu} / \mathrm{K} \alpha$ radiation in the $2 \theta$ range of $5^{\circ}-70^{\circ}$. The preferred orientation of the films was determined using two-dimensional wide-angle X-ray diffraction (2D-WAXD) ring patterns, which were obtained using a transmission geometry X-ray diffractometer (Kappa ApexII Duo, Bruker AXS GmbH, Karlsruhe, Germany). The surface morphology of the films was studied using a scanning electron microscope (SEM) (FEI Inspect-F, Hillsboro, OR, USA). Before gold coating, samples were etched in potassium permanganate solution for $40 \mathrm{~min}$ at $50{ }^{\circ} \mathrm{C}$ to remove the amorphous region. The thermal properties of the materials were analyzed using a differential scanning calorimeter (DSC) (DSC822e, Mettler-Toledo, $\mathrm{OH}, \mathrm{USA}$ ) under a $\mathrm{N}_{2}$ atmosphere. All of the samples were initially heated up to $180{ }^{\circ} \mathrm{C}$ and kept at this temperature for $5 \mathrm{~min}$, then cooled down to $25{ }^{\circ} \mathrm{C}$ and heated up again to $180{ }^{\circ} \mathrm{C}$. Both the heating and cooling rates were set to $5{ }^{\circ} \mathrm{C} \mathrm{min}^{-1}$. Isothermal crystallization was carried out at $150{ }^{\circ} \mathrm{C}$ and $135^{\circ} \mathrm{C}$. The frequency dependence of dielectric permittivity and dielectric loss tangent was measured using a Precision Impedance Analyzer (4294A, Agilent, Santa Clara, CA) at ambient temperature in the frequency range of $100 \mathrm{~Hz}$ to $100 \mathrm{MHz}$ at an applied maximum voltage of $0.5 \mathrm{~V}$. The temperature dependence of dielectric permittivity and dielectric loss tangent was measured at different frequencies using a LCR meter (4284A, Agilent, Santa Clara, CA) which is connected to a homemade furnace. The electrode diameter for the dielectric tests was $5 \mathrm{~mm}$. The ferroelectric $P-E$ hysteresis loops were tested using a ferroelectric hysteresis measurement tester (NPL, Teddington, UK) at ambient temperature and $10 \mathrm{~Hz}$. The electrode diameter for ferroelectric tests was $2 \mathrm{~mm}$. Both the dielectric and ferroelectric data presented in this paper were based on the testing of 8 different specimens.

\section{Results and discussion}

Crystalline phases and the preferred orientation of PVDF/PVDFTrFE blended films

The FTIR spectra of the blended films are shown in Fig. 1a. For pure PVDF, the strong $\alpha$-phase characteristic bands at 1211, 1179, 1145, 1066, 976, 871, 854, 795, 764 and $613 \mathrm{~cm}^{-1}$ can be seen in line (1). FTIR cannot clearly distinguish the $\beta$-phase from the $\gamma$-phase since several of their characteristic bands overlap. $^{23,24}$ For example, the typical $840 \mathrm{~cm}^{-1} \beta$-phase band could also be a superposition of bands for the $\beta$ - and $\gamma$-phases. ${ }^{24,25}$ However, the exclusive $\gamma$-phase bands at 1234, 1117, 833 and $812 \mathrm{~cm}^{-1}$ are not apparent in line (1), ${ }^{26}$ which means that only the $\beta$-phase contributed to the formation of the band at $840 \mathrm{~cm}^{-1}$. To sum up, pure PVDF films mainly crystallized into the $\alpha$-phase with a small amount of the $\beta$-phase ( $\sim 8$ wt $\%$ as shown in Fig. 1b). 

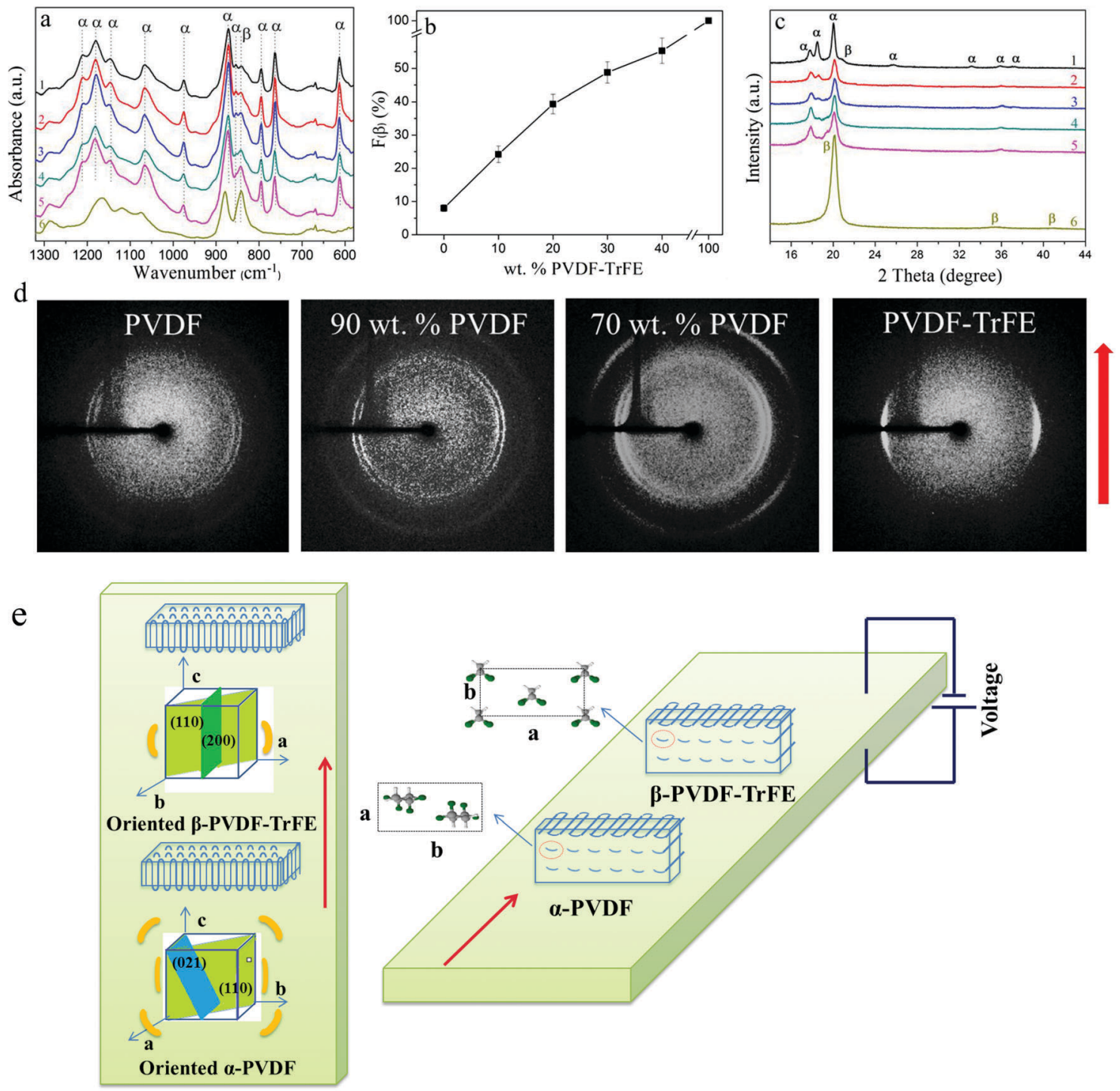

Fig. 1 (a) FTIR spectra of: (1) PVDF/PVDF-TrFE 100/0; (2) PVDF/PVDF-TrFE 90/10 wt\%; (3) PVDF/PVDF-TrFE 80/20 wt\%; (4) PVDF/PVDF-TrFE 70/30 wt\%; (5) PVDF/PVDF-TrFE 60/40 wt\%; and (6) PVDF/PVDF-TrFE 0/100; (b) $F(\beta)$ of pure PVDF and blended films as a function of wt\% PVDF-TrFE; (c) 1D-WAXD patterns of: (1) PVDF/PVDF-TrFE 100/0; (2) PVDF/PVDF-TrFE 90/10 wt\%; (3) PVDF/PVDF-TrFE 80/20 wt\%; (4) PVDF/PVDF-TrFE 70/30 wt\%; (5) PVDF/ PVDF-TrFE 60/40 wt\%; and (6) PVDF/PVDF-TrFE 0/100; (d) 2D-WAXD patterns of PVDF, PVDF-TrFE and blends containing 90 wt\% and 70 wt\% PVDF; and (e) schematic diagram illustrating the orientation of the blended films; the left reflects the film surface and can be used for understanding the 2D-WAXD pattern; the right reflects the cross-section region. The rectangles represent the lamellae with folded polymer chains. The red arrows indicate the extrusion direction.

For pure PVDF-TrFE, strong characteristic $\beta$-phase bands at 1167,878 and $840 \mathrm{~cm}^{-1}$ can be seen in Fig. 1a line (6). The blended films show a mixture of $\alpha$ - and $\beta$-phases. The intensity of the $854 \mathrm{~cm}^{-1}$ band ( $\alpha$-phase) was considerably reduced with increasing amount of PVDF-TrFE, while the $840 \mathrm{~cm}^{-1}$ band ( $\beta$-phase) became more obvious. Eqn (1) was used to quantify the relative fraction of the $\beta$-phase $(F(\beta))$, assuming that only the $\alpha$ - and $\beta$-phases existed. ${ }^{27,28}$ Eqn (1) is built on the assumption that FTIR follows the Lambert-Beer law. ${ }^{27}$ In Eqn (1), $A_{\alpha}$ and $A_{\beta}$ correspond to the measured absorbance at $764 \mathrm{~cm}^{-1}$ and $840 \mathrm{~cm}^{-1}$, and $K_{\alpha}$ and $K_{\beta}$ are the absorbance coefficients at $764 \mathrm{~cm}^{-1}$ and $840 \mathrm{~cm}^{-1}$, the values of which are $6.1 \times 10^{4}$ and $7.7 \times 10^{4} \mathrm{~cm}^{2} \mathrm{~mol}^{-1}$, respectively. ${ }^{27}$ The values of $F(\beta)$ for the blended films are shown in Fig. 1b; the values of $F(\beta)$ of PVDF and PVDF-TrFE are also included. The $F(\beta)$ value increases to almost $40 \mathrm{wt} \%$ for PVDF/PVDF-TrFE $80 / 20 \mathrm{wt} \%$ blended films, which shows that the introduction of PVDF-TrFE promotes the crystallization of PVDF into the $\beta$-phase.

$$
F(\beta)=\frac{A_{\beta}}{\left(\frac{K_{\beta}}{K_{\alpha}}\right) A_{\alpha}+A_{\beta}}
$$

Fig. 1c shows the 1D-WAXD patterns of the pure PVDF, PVDFTrFE, and blended films. The three main peaks of the pure PVDF films, at $2 \theta=17.82^{\circ}, 18.48^{\circ}$ and $20.05^{\circ}$, suggest that PVDF mainly crystallized into the $\alpha$-phase, ${ }^{10,29-34}$ consistent with the 
FTIR data which shows no traces of the $\gamma$-phase but about $8 \mathrm{wt} \%$ of the $\beta$-phase. The shoulder peak of PVDF at $2 \theta=20.08^{\circ}$ also indicates the existence of a small amount of $\beta$-phase in pure PVDF films. For the pure PVDF-TrFE films, one strong $(110) /(200)$ reflection peak at $2 \theta=20.12^{\circ}$ was observed. Two other peaks at $2 \theta=35.5^{\circ}$ and $40.9^{\circ}$ were extremely weak and broad, which indicates a highly preferred orientation for the pure PVDF-TrFE films. ${ }^{33}$

Similar to PVDF, the blended films showed three XRD peaks. The intensity of the characteristic (020) $\alpha$-PVDF peak at about $18.5^{\circ}$ significantly reduced with the PVDF-TrFE content, especially for the blended films containing more than $20 \mathrm{wt} \%$ PVDF-TrFE. The weakening of this peak indicates that the amount of $\alpha$-phase was reduced and/or the preferred orientation of the crystallites increased with the presence of PVDF-TrFE. Combined with the FTIR data, it can be confirmed that there was a reduction in the $\alpha$-phase and a corresponding increase in the $\beta$-phase PVDF.

The preferred orientation results of the PVDF and PVDFTrFE films obtained from 2D-WAXD analysis are shown in Fig. 1d. From inner to outer, the WAXD reflections of the PVDF, calculated from Fig. 1d, are $18.1^{\circ}, 20.0^{\circ}$ and $26.6^{\circ}$. The ring at $18.1^{\circ}$ consists of the overlapping $17.81^{\circ}(100)_{\alpha}$ and $18.48^{\circ}(020)_{\alpha}$ reflections. The reflection at $26.6^{\circ}$, though not obvious in Fig. 1c, is associated with the $(021)_{\alpha}$ plane, which is characteristic of the $\alpha$-phase. As clearly seen in Fig. 1d, the crystalline phase of PVDF-TrFE is well oriented, with the $(110) \beta /(200) \beta$
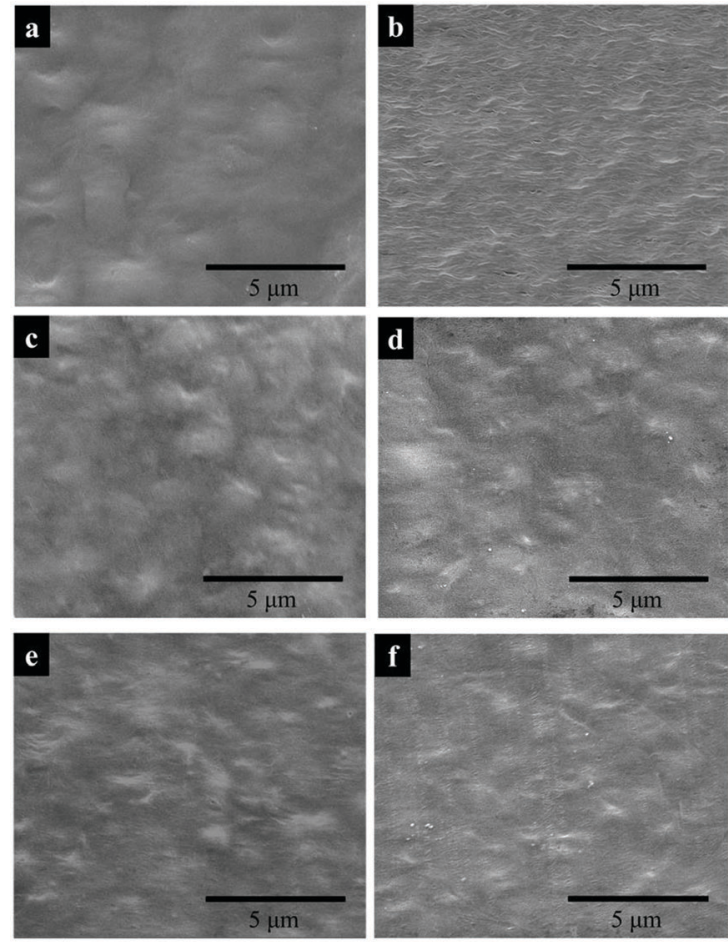

Fig. 2 SEM images of surfaces of: (a) PVDF; (b) PVDF-TrFE; (c) PVDF/ PVDF-TrFE 90/10 wt\%; (d) PVDF/PVDF-TrFE 80/20 wt\%; (e) PVDF/PVDFTrFE 70/30 wt\%; and (f) PVDF/PVDF-TrFE 60/40 wt\% (arrow indicates the extrusion direction). reflections concentrated towards the equatorial region, indicating that the polymer chain axis (c-axis) is oriented parallel to the extrusion direction. ${ }^{21}$ In comparison, the reflections of PVDF are more uniformly distributed, implying a low preferred orientation. The orientation difference can be explained by the fact that PVDF-TrFE exhibits a longer relaxation time in the melt state than that of PVDF, and therefore showed a more pronounced crystal orientation during flow extension. ${ }^{35}$

The 2D-WAXD patterns of the blended films are shown in Fig. 1d. From inner to outer, the WAXD profiles exhibit the
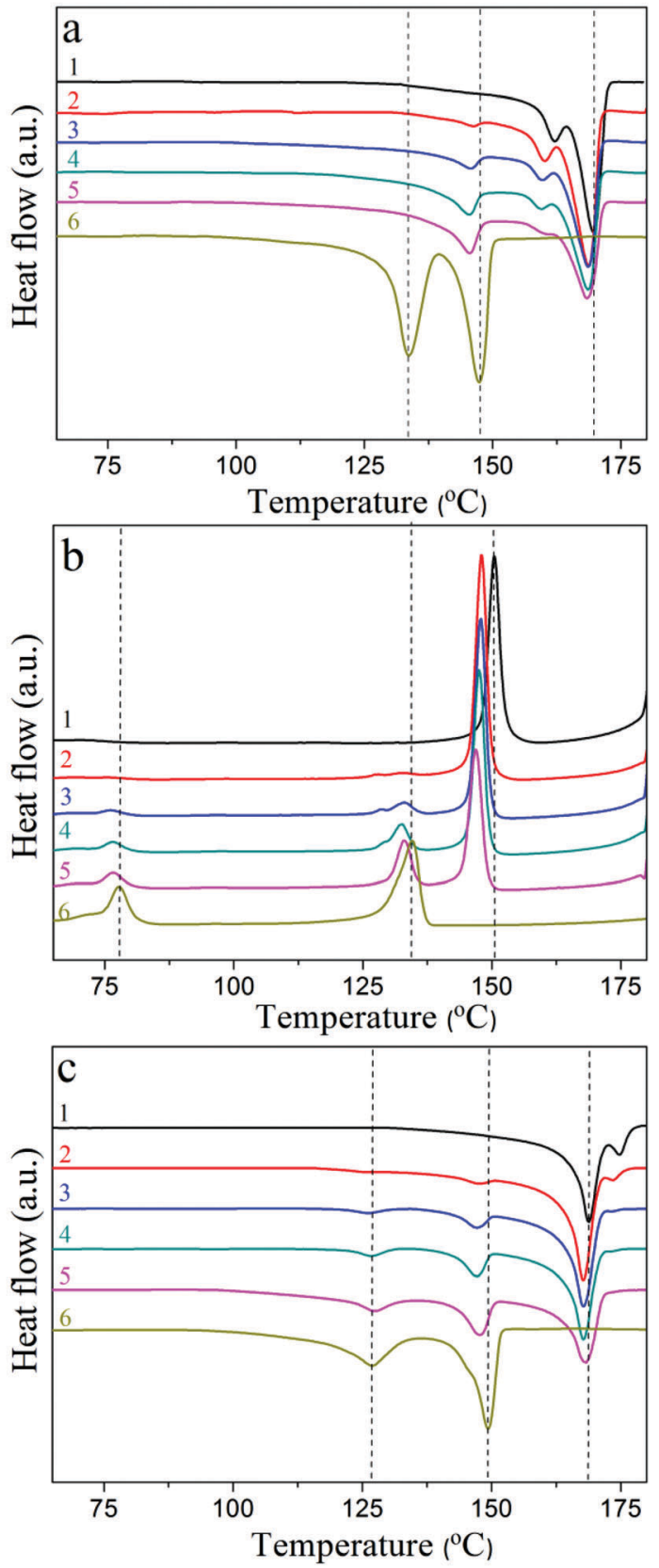

Fig. 3 (a) First heating; (b) cooling; and (c) second heating DSC graphs of: (1) PVDF/PVDF-TrFE 100/0; (2) PVDF/PVDF-TrFE 90/10 wt\%; (3) PVDF/ PVDF-TrFE 80/20 wt\%; (4) PVDF/PVDF-TrFE 70/30 wt\%; (5) PVDF/PVDFTrFE 60/40 wt\%; and (6) PVDF/PVDF-TrFE 0/100. 
Table 1 The enthalpy of Curie transition and the fusion of PVDF/PVDF-TrFE blended films acquired from first heating and second heating DSC curves

\begin{tabular}{|c|c|c|c|c|c|c|}
\hline PVDF/PVDF-TrFE & \multicolumn{3}{|c|}{ Enthalpy values of first heating $\left(\mathrm{J} \mathrm{g}^{-1}\right)$} & \multicolumn{3}{|c|}{ Enthalpy values of second heating $\left(\mathrm{J} \mathrm{g}^{-1}\right)$} \\
\hline $100 / 0$ & - & - & $46.3 \pm 3$ & - & - & $43.1 \pm 2$ \\
\hline $80 / 20$ & - & $6.6 \pm 1$ & $36.4 \pm 3$ & $9.0 \pm 1$ & $13.6 \pm 1$ & $32.7 \pm 1$ \\
\hline $70 / 30$ & - & $17.1 \pm 2$ & $33.0 \pm 3$ & $7.8 \pm 1$ & $19.6 \pm 1$ & $32.0 \pm 1$ \\
\hline $60 / 40$ & - & $15.6 \pm 1$ & $32.5 \pm 2$ & $12.2 \pm 2$ & $21.0 \pm 1$ & $31.9 \pm 1$ \\
\hline
\end{tabular}

${ }^{a} \Delta H_{\mathrm{c} \text { PVDF-TrFE: }}$ enthalpy of Curie transition of PVDF-TrFE; $\Delta H_{\mathrm{fPVDF}-T r F E}$ and $\Delta H_{\mathrm{f} \mathrm{PVDF}}$ : fusion enthalpy of PVDF-TrFE and PVDF, respectively.

characteristic reflections of $(100)_{\alpha} /(020)_{\alpha},(110)_{\alpha+\beta} /(200)_{\beta}$ and $(021)_{\alpha}$ planes at $18.36^{\circ}, 20.33^{\circ}$ and $26.6^{\circ}$ respectively. With increasing amount of PVDF-TrFE, the preferred orientation of the $(110)_{\alpha+\beta} /(200)_{\beta}$ and $(021)_{\alpha}$ reflections is enhanced. The intensity as a function of the azimuthal angle from $-90^{\circ}$ to $+90^{\circ}$ at the radial position of the $(110)_{\alpha+\beta} /(200)_{\beta}$ and $(100)_{\alpha} /(020)_{\alpha}$ peaks for the pure PVDF, PVDF-TrFE and blended films was fitted with a Gaussian function (Fig. S1, ESI $\dagger$ ). Pure PVDF films show the least preferred orientation, corresponding to the broadest peak (Fig. S1, ESI $\dagger$ ). For the blended films (Fig. S1, ESI $\dagger$ ) the intensity is enhanced and the peak becomes sharper radially and azimuthally with increasing amount of PVDF-TrFE, which shows that blending with PVDF-TrFE leads to increased crystallinity and higher preferred orientation for the PVDF/PVDF-TrFE blended films. Interestingly, the outermost $26.6^{\circ}(021)_{\alpha}$ reflection ring of the blended films shows a preferred orientation, about $45^{\circ}$ from the equatorial direction, which enhanced with increasing amount of PVDF-TrFE (Fig. 1d). During extrusion, the temperature dropped quickly from $205{ }^{\circ} \mathrm{C}$ to room temperature, which caused the PVDF and PVDF-TrFE to crystallize simultaneously. The existence of a strong interaction between the two different polymers caused the chains of the PVDF to orientate in the same direction as the PVDF-TrFE. Fig. 1e depicts the orientation of the crystallites of the blended films formed during the extrusion process.

\section{Miscibility and crystallization behavior of PVDF/PVDF-TrFE blended films}

Morphology studies. It is known that PVDF crystallizes as spherulites when prepared by melt processing, ${ }^{7}$ while PVDFTrFE crystallizes with a stacked lamellar structure ${ }^{36}$ Fig. $2 \mathrm{a}$ and b shows the surface morphology of the PVDF and PVDF-TrFE films. The arrow in Fig. 2 indicates the extrusion direction. For PVDF, the lamellae tend to form spherulites with little preferred orientation, while PVDF-TrFE displays a stacked lamellar morphology. ${ }^{37}$ For the blended films, the two components crystallize together without obvious phase separation (Fig. 2c-f). Furthermore, the introduction of PVDF-TrFE produces distortion of the spherulites as can be seen in Fig. $2 \mathrm{f}$ for the PVDF/PVDFTrFE 60/40 wt $\%$ blended film.

Thermal analysis. Fig. 3 shows the DSC scans of PVDF, PVDF-TrFE and their blends. The first heating curves are displayed in Fig. 3a. Pure PVDF has two obvious fusion peaks at $161.3 \pm 0.3{ }^{\circ} \mathrm{C}$ and $169.9 \pm 0.4{ }^{\circ} \mathrm{C}$, which could be caused by the existence of different crystalline phases or crystallization imperfection. The melting endotherms of $\alpha$ - and $\beta$-PVDF are reported to be almost at the same position, both at around $167{ }^{\circ} \mathrm{C}^{27}$ Combined with the FTIR results of PVDF, it can be deduced that the $169.9^{\circ} \mathrm{C}$ endotherm peak corresponds to the melting of the well-formed prevalent $\alpha$-phase crystals, while the $161.3{ }^{\circ} \mathrm{C}$ peak can be attributed to the melting of imperfect crystalline regions. ${ }^{38,39}$ It is seen in Table 1 that pure PVDF exhibits a fusion enthalpy $\left(\Delta H_{\mathrm{f}}\right)$ of $46.3 \mathrm{~J} \mathrm{~g}^{-1}$, indicating that the crystallinity of the pure PVDF extruded films is about $44 \%$ $\left(\Delta H_{\mathrm{f}}\right.$ for $100 \%$ crystalline PVDF is $\left.104.6 \mathrm{~J} \mathrm{~g}^{-140}\right)$.
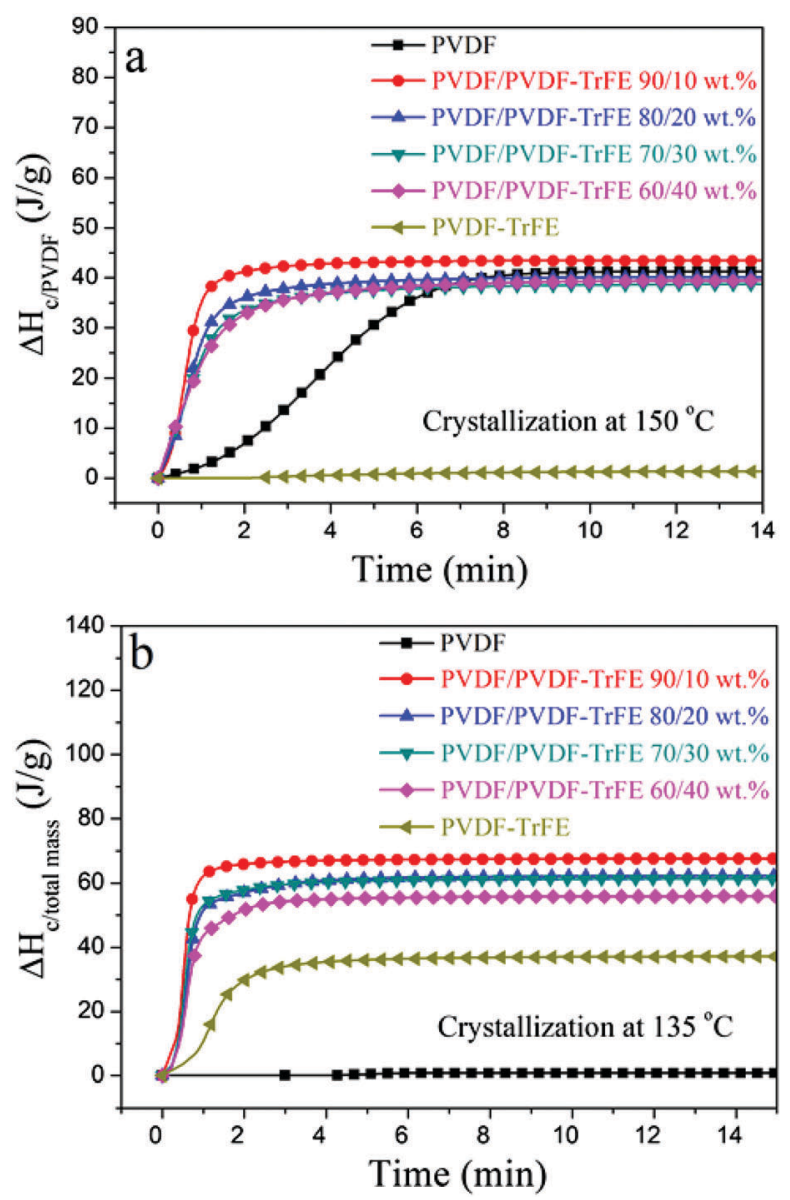

Fig. 4 Crystallization enthalpy $\left(\Delta H_{\mathrm{c}}\right)$ acquired by integrating heat flow recorded during isothermal crystallization as a function of time at: (a) $150{ }^{\circ} \mathrm{C}$ and (b) $135{ }^{\circ} \mathrm{C}$. The $\Delta H_{\mathrm{c}}$ values at $150{ }^{\circ} \mathrm{C}$ for blend materials were normalized by PVDF. 
a

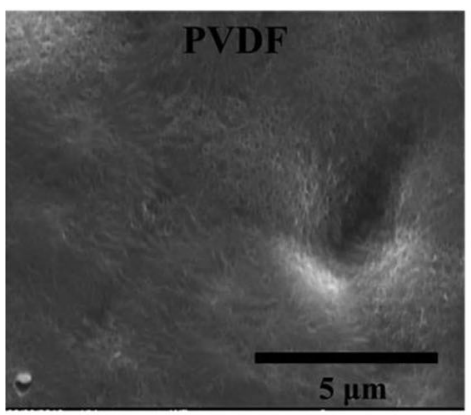

b

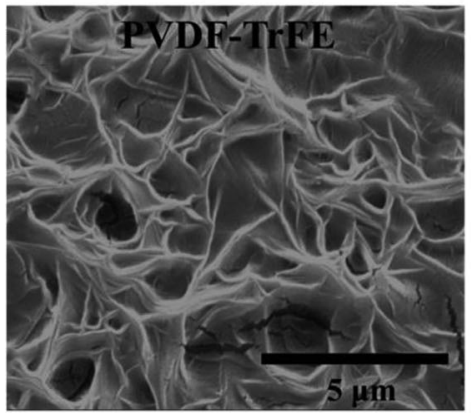

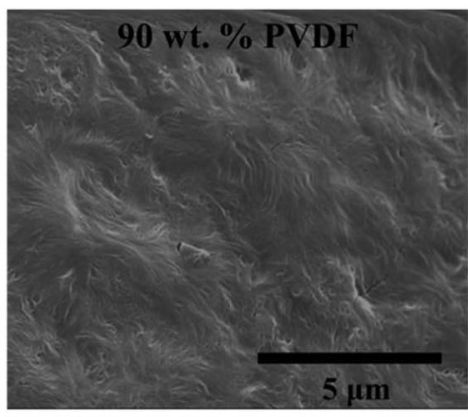

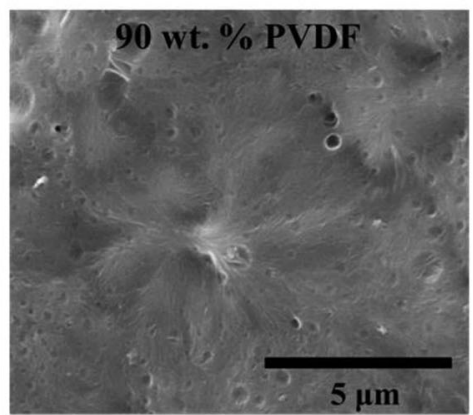

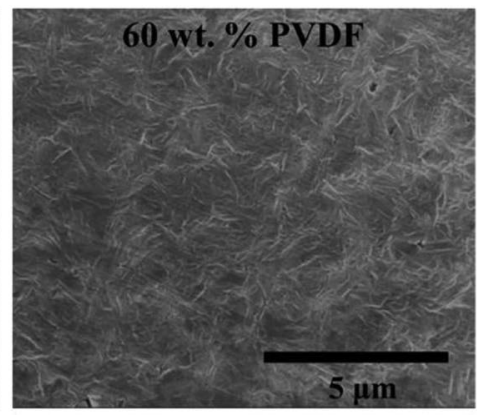

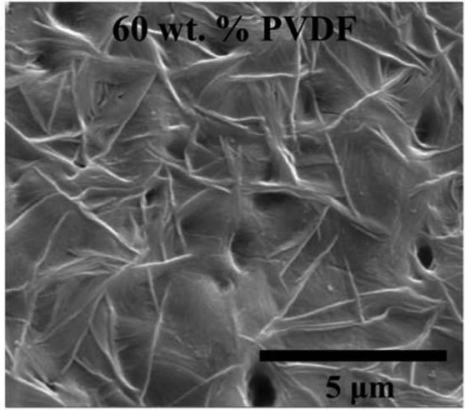

Fig. 5 SEM morphology images of samples isothermally crystallized at (a) $150{ }^{\circ} \mathrm{C}$ and (b) $135^{\circ} \mathrm{C}$.

Apart from its fusion peak at 147.2 $\pm 0.2{ }^{\circ} \mathrm{C}$, pure PVDF-TrFE shows another peak at $133.8 \pm 0.1{ }^{\circ} \mathrm{C}$ originating from the ferroelectric to paraelectric phase transition (Curie transition). The $\Delta H_{\mathrm{f}}$ of PVDF-TrFE is $29.0 \mathrm{~J} \mathrm{~g}^{-1}$, suggesting a crystallinity of $76 \%\left(\Delta H_{\mathrm{f}}\right.$ for $100 \%$ crystalline PVDF-TrFE is about $\left.38 \mathrm{~J} \mathrm{~g}^{-141}\right)$. The blended films exhibit three peaks on first heating, corresponding to the fusion peaks of PVDF-TrFE and PVDF, which proves the immiscibility of the two polymers. Interestingly, the Curie transition peak of PVDF-TrFE is diffuse and is apparent only as a shoulder on the lower temperature side of the fusion peak of PVDF-TrFE.

Fig. $3 \mathrm{~b}$ shows the cooling DSC curves on cooling after first heating. Pure PVDF has one crystallization peak at $150.6 \pm$ $0.5{ }^{\circ} \mathrm{C}$, and pure PVDF-TrFE shows two peaks at $134.6 \pm 0.8^{\circ} \mathrm{C}$ and $77.8 \pm 0.5{ }^{\circ} \mathrm{C}$, resulting from the crystallization and the paraelectric to ferroelectric phase transition, respectively. All blended films exhibit three peaks. The crystallization temperatures of PVDF and PVDF-TrFE and the Curie transition in the blended films are slightly lower than those of the pure components.

Fig. $3 c$ shows the second heating DSC curves of PVDF, PVDFTrFE and their blends. During the second heating pure PVDF has two fusion peaks at $168.9 \pm 0.3{ }^{\circ} \mathrm{C}$ and $174.8 \pm 0.2{ }^{\circ} \mathrm{C}$, indicating a mixture of $\alpha$ - and $\gamma$-phases. ${ }^{23}$ Further evidence for the presence of the $\gamma$-phase can be found in the FTIR data presented in Fig. S2 (ESI $\dagger$ ). With regard to pure PVDF-TrFE, the peak value of the Curie transition shifts to a lower temperature $\left(127 \pm 0.1^{\circ} \mathrm{C}\right)$ when compared to the first heating $\left(133.8 \pm 0.1^{\circ} \mathrm{C}\right)$. The higher Curie point in the first heating indicates that the pure PVDF-TrFE crystallized into highly oriented ferroelectric crystals through the extrusion method. ${ }^{42}$ For the blended films, the Curie transition peak was diffuse in the first heating curves, however, a small, but clear, peak can be seen in the second
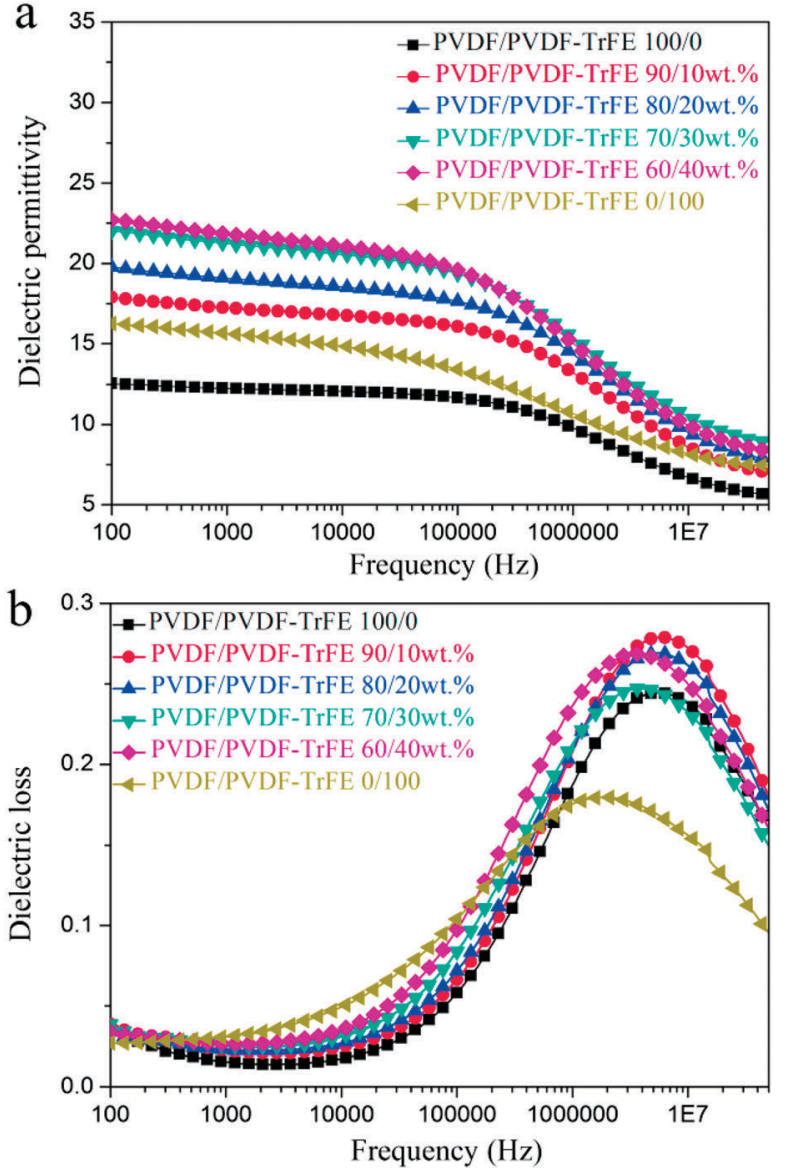

Fig. 6 Frequency dependence of (a) dielectric permittivity and (b) dielectric loss for pure PVDF, PVDF-TrFE and blended films as a function of frequency. 
heating curves. It is shown in Table 1 that $\Delta H_{\mathrm{fPVDF}}$ of pure PVDF and blended samples during first heating are larger than those of second heating, while being lower for $\Delta H_{\mathrm{f} \text { PVDF-TrFE }}$, which indicates the existence of interactions between PVDF and PVDF-TrFE in the extruded blended films. PVDF crystallized first and served as a nucleating agent in the crystallization of PVDF-TrFE. The formed crystallites exhibited similar structures and were intimately correlated. The Curie transition is achieved by the formation of gauche bonds, which requires the polymer chains in PVDF-TrFE to undergo severe twisting and/or tilting, ${ }^{42}$ which needs adequate space to accomplish this. However, the surrounding PVDF crystals and the intimate coexistence of the two components restricts the space to accomplish the transition. However, PVDF and PVDF-TrFE crystallized more freely during the DSC slow cooling process (cooling rate $5{ }^{\circ} \mathrm{C} \mathrm{min}^{-1}$ ), which resulted in more phase separation and less interactions, making the Curie transition peaks more obvious than those of the extruded blended films in the first DSC heating curves.

The above results, correlated with both crystallization and morphological studies, strongly demonstrate the intimate interactions between the PVDF and PVDF-TrFE. More detailed investigations of isothermal crystallization at $150{ }^{\circ} \mathrm{C}$ (PVDF crystallization temperature) and $135{ }^{\circ} \mathrm{C}$ (PVDF-TrFE crystallization temperature) were undertaken.

The DSC data recorded during isothermal crystallization at $150{ }^{\circ} \mathrm{C}$ are shown in Fig. $4 \mathrm{a}$ and Fig. S3 (ESI $\dagger$ ), and the morphology of the films is shown in Fig. 5a. No crystallization of PVDF-TrFE occurred at $150{ }^{\circ} \mathrm{C}$ (Fig. S3, ESI $\dagger$ ). It is evident that the rate of crystallization of PVDF at $150{ }^{\circ} \mathrm{C}$ was increased by the addition of PVDF-TrFE (in the melt state). This is different to what is reported for PVDF/poly(1,4-butylene adipate) (PBA) blends where the crystallization rate of PVDF was reduced due to the presence of PBA. ${ }^{43}$ To understand these differences, it is necessary to consider the morphologies of the microstructures. In the PVDF/PBA system, PVDF crystallized into progressively larger spherulites with increasing PBA content, however, in our PVDF/PVDF-TrFE blends the growth of PVDF spherulites was restricted. The isothermally crystallized PVDF showed fine spherulites. The spherulites that formed in the blended samples were smaller and less perfect compared to those in PVDF (Fig. 5a), which is consistent with the morphology of extruded films. On the basis that there was no crystallization of PVDF-TrFE at $150{ }^{\circ} \mathrm{C}$ because the temperature was above its melting point, the crystallization enthalpy of the blends was normalized in terms of the PVDF content $\left(\Delta H_{\mathrm{c} / \mathrm{PVDF}}\right)$. Fig. $4 \mathrm{a}$ shows that the normalized values of $\Delta H_{\mathrm{c} / \mathrm{PVDF}}$ for both pure PVDF and the blends are similar regardless of the weight ratio, indicative of almost no hindrance to the degree of crystallinity of PVDF due to the introduction of PVDF-TrFE.

The non-normalized raw DSC data for samples isothermally crystallized at $135{ }^{\circ} \mathrm{C}$ are shown in Fig. 4b and Fig. S3 (ESI $\dagger$ ). Pure PVDF-TrFE exhibited a maximum $\Delta H_{\mathrm{c}}$ of approximately $37 \mathrm{~J} \mathrm{~g}^{-1}$, which represents almost complete crystallization with a reported enthalpy of $100 \%$ crystalline PVDF-TrFE $\left(\sim 38 \mathrm{~J} \mathrm{~g}^{-1}\right) .^{41}$
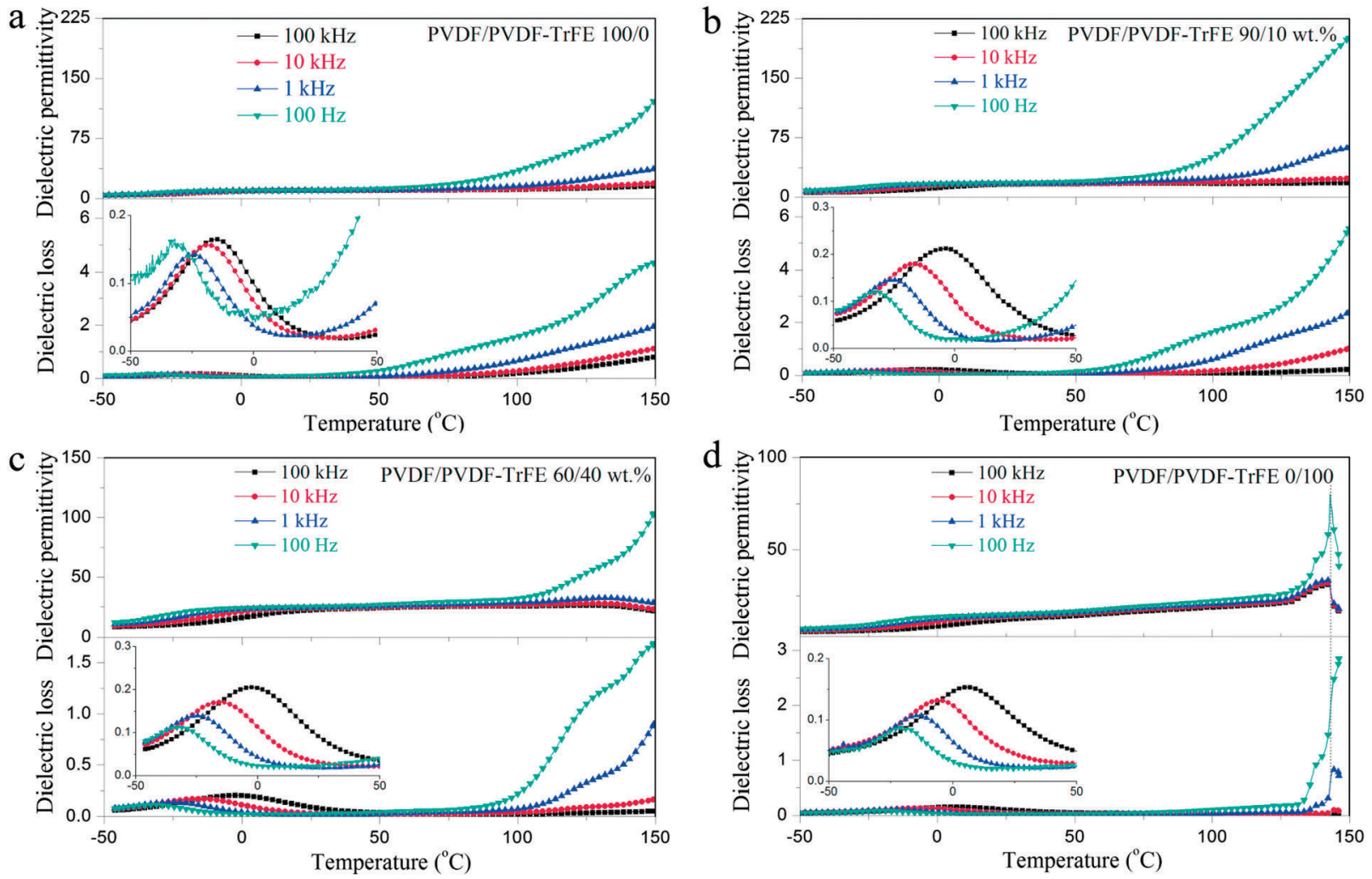

Fig. 7 Temperature dependence of the dielectric permittivity and loss of: (a) PVDF; (b) PVDF/PVDF-TrFE 90/10 wt\%; (c) PVDF/PVDF-TrFE 60/40 wt\%; and (d) PVDF-TrFE. 
During the isothermal crystallization at $135{ }^{\circ} \mathrm{C}$, PVDF continued to crystallize as demonstrated by the large enthalpies of the blends. On the other hand, the rate of crystallization at $135{ }^{\circ} \mathrm{C}$ of PVDF-TrFE was increased in the blends compared to the pure copolymer. This can be explained by the PVDF crystallites acting as nucleation sites for the crystallization of PVDF-TrFE. Fig. 5b shows the morphology of samples that isothermally crystallized at $135{ }^{\circ} \mathrm{C}$, with needle-like PVDF-TrFE crystals embedded in the matrix of PVDF, which did not crystallize into a spherulitic structure. To conclude, the DSC data (Fig. 3, 4 and Table 1) and the microstructural analysis (Fig. 2 and 5) clearly show that synergistic effects occurred at the nanoscale in the blended materials at the interface between the two immiscible polymers that strongly affected the kinetics of crystallization and the microstructures that formed.

\section{Electric properties of PVDF/PVDF-TrFE blended films}

Dielectric properties. Fig. $6 \mathrm{a}$ and $\mathrm{b}$ shows the frequency dependence of the dielectric permittivity and the loss of PVDF, PVDF-TrFE and their blends. The blended films show larger dielectric constant values than those of the two pure components. One explanation for this could be enhanced interfacial polarization at the PVDF and PVDF-TrFE interfaces. Another possible explanation might be the preferred orientation of the polymer chains in the amorphous region, especially at the crystallineamorphous interfaces. ${ }^{44}$ The addition of PVDF-TrFE in the blended films increased the crystalline preferred orientation of the PVDF, thus causing the chains in the amorphous region to orient along the same direction, which gives rise to higher dielectric constant values than for pure PVDF.

Fig. 7 shows the temperature dependent dielectric spectra of pure PVDF, PVDF-TrFE and their blends. All of the samples exhibit a dielectric loss peak at about $0{ }^{\circ} \mathrm{C}$, which is ascribed to the relaxation of the polymer chains in the amorphous regions (glass transition). ${ }^{5}$ The dielectric permittivity of PVDF-TrFE shows an obvious peak at about $140{ }^{\circ} \mathrm{C}$, and the peak position is frequency invariant, which suggests the existence of a Curie transition. ${ }^{45}$ The blended films with $40 \mathrm{wt} \%$ PVDF-TrFE show an inflexion in the permitivity and loss data consistent with a Curie transition (Fig. 7c).

Ferroelectric properties. Fig. 8 shows the ferroelectric polarization hysteresis loops for PVDF, PVDF-TrFE and the blended films. The $P-E$ loops are saturated, which was confirmed by the invariance of the current peak position beyond a certain maximum applied electric field $\left(\sim 120 \mathrm{kV} \mathrm{mm}^{-1}\right)$. The PVDFTrFE exhibits good ferroelectric properties, with a coercive field of about $35 \mathrm{kV} \mathrm{mm}^{-1}$ and a remnant polarization of $0.09 \mathrm{C} \mathrm{m}^{-2}$, which are attributed to its highly preferred crystalline orientation and high crystallinity. ${ }^{21}$ In the case of PVDF, a ferroelectric response was observed, confirmed by the presence of weak current peaks. The ferroelectric behavior of PVDF is attributed to the presence of a small amount of $\beta$-phase $(\sim 8 \mathrm{wt} \%)$ and the transformation of the paraelectric $\alpha$-phase into the ferroelectric $\delta$-phase during the measurement.

With regard to the blended films, the coercive field decreased from $83 \mathrm{kV} \mathrm{mm}{ }^{-1}$ to $32 \mathrm{kV} \mathrm{mm}{ }^{-1}$ with increasing amount of
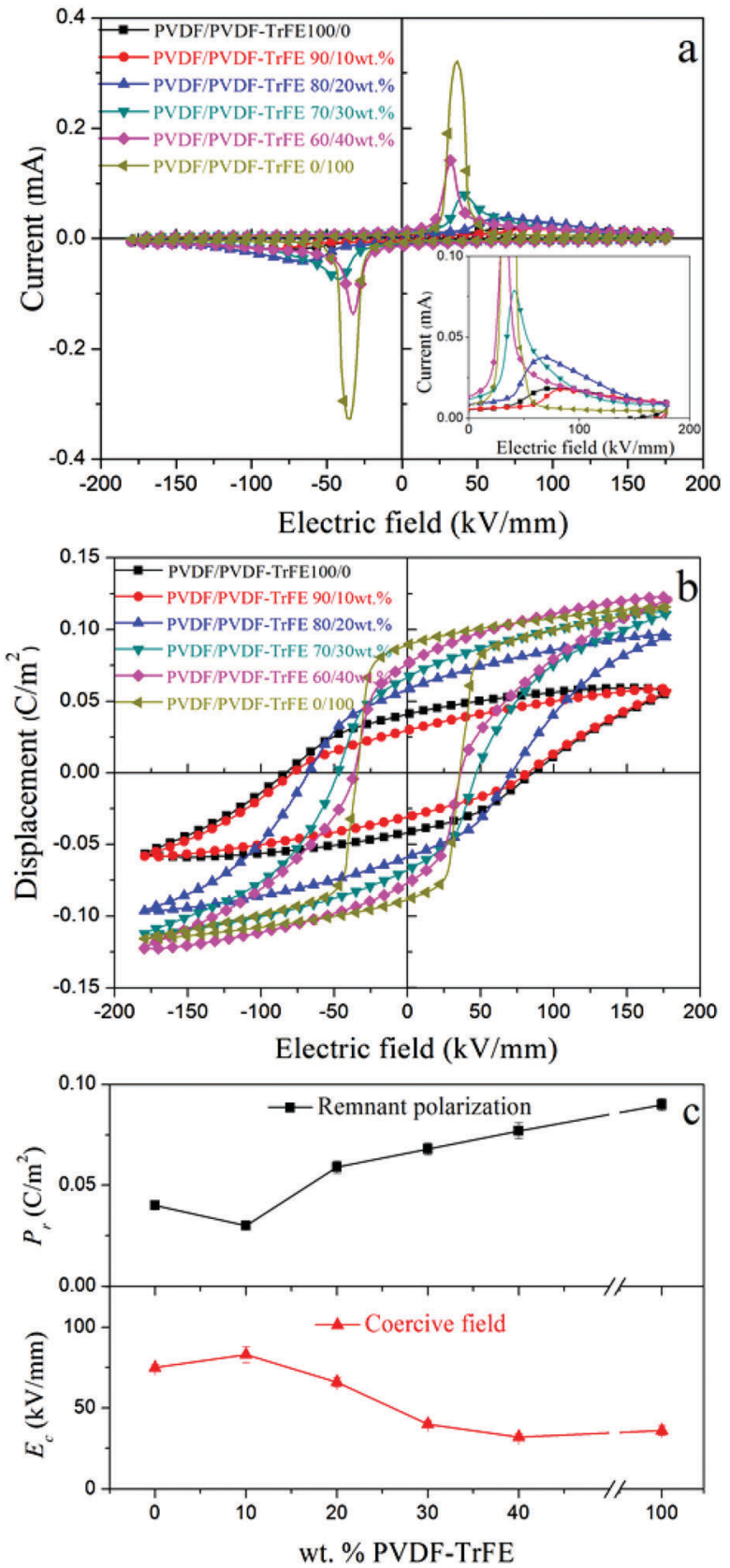

Fig. 8 Ferroelectric properties of: (1) PVDF/PVDF-TrFE 100/0; (2) PVDF/ PVDF-TrFE 90/10 wt\%; (3) PVDF/PVDF-TrFE 80/20 wt\%; (4) PVDF/PVDFTrFE 70/30 wt\%; (5) PVDF/PVDF-TrFE 60/40 wt\%; and (6) PVDF/PVDFTrFE 0/100: (a) current-electric field I-E curves; (b) polarization-electric field $P-E$ loops; (c) variations of remnant polarization $P_{\mathrm{r}}$ and switching field $E_{\mathrm{c}}$ as a function of wt\% PVDF-TrFE (data collected at $E=180 \mathrm{kV} \mathrm{mm}^{-1}$ ).

PVDF-TrFE from $10 \mathrm{wt} \%$ to $40 \mathrm{wt} \%$ as a result of blending. The remnant polarization of the PVDF is apparently higher compared with $10 \mathrm{wt} \%$ PVDF-TrFE due to a leakage current in pure PVDF, making the remnant polarization of PVDF unrealistically high (inset in Fig. 8a). The leakage current could be ascribed to the gaps or voids formed between large PVDF spherulites. ${ }^{46}$ The introduction of PVDF-TrFE enhanced the remnant polarization for the blended films from 0.030 to $0.077 \mathrm{C} \mathrm{m}^{-2}$ with increasing 
the amounts of PVDF-TrFE from 10 to $40 \mathrm{wt} \%$ (Fig. 8c). On the basis of the data from the structural characterization, the addition of PVDF-TrFE enhanced the crystallization of the ferroelectric $\beta$-phase in the blended films, but this alone would not explain the enhanced ferroelectric properties of the blended films.

The theoretical value of the remnant polarization of the blended film with $40 \mathrm{wt} \%$ copolymer based on the simple mixing rule was calculated using the following equation: $P_{\text {rblends }}=\varphi_{\mathrm{PVDF}} \times P_{\mathrm{rPVDF}}+\varphi_{\mathrm{PVDF}-\mathrm{TrFE}} \times P_{\mathrm{rPVDF}-\mathrm{TrFE}}$, where $\varphi$ and $P_{\mathrm{r}}$ are the volume fraction and the measured remnant polarization of pure PVDF and PVDF-TrFE extruded samples. The calculated value for the blended film with $40 \mathrm{wt} \%$ copolymer is only $0.058 \mathrm{C} \mathrm{m}^{-2}$, about $25 \%$ lower than the experimental value. Similar conditions existed in the $20 \mathrm{wt} \%$ and $30 \mathrm{wt} \%$ blends, where the calculated values were about $20 \%$ less compared with the experimental values. Combined with the diffuse Curie transition and larger dielectric constants observed for the blended films, the interaction between the two polymers and the interfaces between them could explain the enhanced ferroelectric properties of the blended films. The interfacial polarization contributed to the higher remnant polarization, and more contributions were generated at high electric fields, as indicated by the large saturated polarization of the blend with $40 \mathrm{wt} \%$ PVDF-TrFE.

\section{Conclusions}

Despite the immiscibility of PVDF and PVDF-TrFE, as demonstrated by the DSC results, they intimately crystallize on a fine scale $(\sim 40 \mathrm{~nm})$ without the appearance of distinct phase separation. The rate of crystallization of PVDF and PVDF-TrFE increased as a result of blending, as suggested by isothermal crystallization studies. With increasing amount of PVDF-TrFE, the blended films had more $\beta$-phase and increased preferred orientation, more than would be expected based on a simple rule of mixtures. Due to interfacial polarization, PVDF/PVDFTrFE blended films had a larger dielectric constant than the two pure components. Furthermore, the ferroelectric properties of the blended films were enhanced more than would be expected based on a simple rule of mixtures. The switching field decreased (from 75 to $32 \mathrm{kV} \mathrm{mm}{ }^{-1}$ ), while the remnant polarization increased (from 0.040 to $0.077 \mathrm{C} \mathrm{m}^{-2}$ ) with increasing amount of PVDF-TrFE from 0 to $40 \mathrm{wt} \%$. The Curie transition was suppressed in the blended films, which may lead to increased high temperature stability for piezoelectric applications.

\section{Acknowledgements}

We appreciate the valuable discussion with Prof Cees Bastiaansen and Prof Ton Peijs. Nan Meng was financially supported by the China Scholarship Council (CSC). Prof Mike Reece would also like to acknowledge the support of The Engineering and Physical Sciences Research Council (EP/L017695/1, MASSIVE).

\section{Notes and references}

1 Y. Yuan, T. J. Reece, P. Sharma, S. Poddar, S. Ducharme, A. Gruverman, Y. Yang and J. Huang, Nat. Mater., 2011, 10, 296-302.

2 M. Li, I. Katsouras, C. Piliego, G. Glasser, I. Lieberwirth, P. W. M. Blom and D. M. de Leeuw, J. Mater. Chem. C, 2013, 1, 7695-7702.

3 B. Li, C. Xu, F. Zhang, J. Zheng and C. Xu, J. Mater. Chem. C, 2015, 3, 8926-8931.

4 A. V. Shirinov and W. K. Schomburg, Sens. Actuators, A, 2008, 142, 48-55.

5 L. Zhu, J. Phys. Chem. Lett., 2014, 5, 3677-3687.

6 Q. Li and Q. Wang, Macromol. Chem. Phys., 2016, 217, 1228-1244.

7 A. J. Lovinger, Science, 1983, 220, 1115-1121.

8 W. M. Prest and D. J. Luca, J. Appl. Phys., 1978, 49, 5042-5047.

9 N. C. Banik, P. L. Taylor and A. J. Hopfinger, Appl. Phys. Lett., 1980, 37, 49-50.

10 G. T. Davis, J. E. McKinney, M. G. Broadhurst and S. C. Roth, J. Appl. Phys., 1978, 49, 4998-5002.

11 K. Matsushige, K. Nagata, S. Imada and T. Takemura, Polymer, 1980, 21, 1391-1397.

12 T. Furukawa, Adv. Colloid Interface Sci., 1997, 71-72, 183-208.

13 O. Hiroji, A. Shuyo and K. Keiko, Jpn. J. Appl. Phys., 1988, 27, 2144.

14 N. Tsutsumi, Y. Ueda, T. Kiyotsukuri, A. S. DeReggi and G. T. Davis, J. Appl. Phys., 1993, 74, 3366-3372.

15 Q. Meng, W. Li, Y. Zheng and Z. Zhang, J. Appl. Polym. Sci., 2010, 116, 2674-2684.

16 Y. Tang and J. Scheinbeim, J. Polym. Sci., Part B: Polym. Phys., 2003, 41, 927-935.

17 M. Li, N. Stingelin, J. J. Michels, M.-J. Spijkman, K. Asadi, K. Feldman, P. W. M. Blom and D. M. de Leeuw, Macromolecules, 2012, 45, 7477-7485.

18 X. Zhang, Y. Shen, Z. Shen, J. Jiang, L. Chen and C.-W. Nan, ACS Appl. Mater. Interfaces, 2016, 8, 27236-27242.

19 H. Tanaka, A. J. Lovinger and D. D. Davis, J. Polym. Sci., Part B: Polym. Phys., 1990, 28, 2183-2198.

20 R. Gregorio, M. R. Chaud, W. Nunes Dos Santos and J. B. Baldo, J. Appl. Polym. Sci., 2002, 85, 1362-1369.

21 N. Meng, R. Mao, W. Tu, X. Zhu, R. M. Wilson, E. Bilotti and M. J. Reece, Polymer, 2016, 100, 69-76.

22 N. Shingne, M. Geuss, B. Hartmann-Azanza, M. Steinhart and T. Thurn-Albrecht, Polymer, 2013, 54, 2737-2744.

23 R. Gregorio, J. Appl. Polym. Sci., 2006, 100, 3272-3279.

24 M. Li, H. J. Wondergem, M.-J. Spijkman, K. Asadi, I. Katsouras, P. W. M. Blom and D. M. de Leeuw, Nat. Mater., 2013, 12, 433-438.

25 R. Gregorio and R. C. CapitãO, J. Mater. Sci., 2000, 35, 299-306.

26 J. H. Park, N. Kurra, M. N. AlMadhoun, I. N. Odeh and H. N. Alshareef, J. Mater. Chem. C, 2015, 3, 2366-2370.

27 J. R. Gregorio and M. Cestari, J. Polym. Sci., Part B: Polym. Phys., 1994, 32, 859-870. 
28 M. P. Silva, V. Sencadas, G. Botelho, A. V. Machado, A. G. Rolo, J. G. Rocha and S. Lanceros-Mendez, Mater. Chem. Phys., 2010, 122, 87-92.

29 M. Sharma, G. Madras and S. Bose, Phys. Chem. Chem. Phys., 2014, 16, 14792-14799.

30 Y. Takahashi and H. Tadokoro, Macromolecules, 1980, 13, 1317-1318.

31 J. Buckley, P. Cebe, D. Cherdack, J. Crawford, B. S. Ince, M. Jenkins, J. Pan, M. Reveley, N. Washington and N. Wolchover, Polymer, 2006, 47, 2411-2422.

32 D. M. Esterly and B. J. Love, J. Polym. Sci., Part B: Polym. Phys., 2004, 42, 91-97.

33 J. L. Lutkenhaus, K. McEnnis, A. Serghei and T. P. Russell, Macromolecules, 2010, 43, 3844-3850.

34 F. Oliveira, Y. Leterrier, J.-A. Månson, O. Sereda, A. Neels, A. Dommann and D. Damjanovic, J. Polym. Sci., Part B: Polym. Phys., 2014, 52, 496-506.

35 P. K. Agarwal, R. H. Somani, W. Weng, A. Mehta, L. Yang, S. Ran, L. Liu and B. S. Hsiao, Macromolecules, 2003, 36, 5226-5235.
36 C. M. Costa, M. N. T. Machiavello, J. L. G. Ribelles and S. Lanceros-Méndez, J. Mater. Sci., 2013, 48, 3494-3504.

37 H. Zhou and G. L. Wilkes, J. Mater. Sci., 1998, 33, 287-303.

38 D. R. Dillon, K. K. Tenneti, C. Y. Li, F. K. Ko, I. Sics and B. S. Hsiao, Polymer, 2006, 47, 1678-1688.

39 J. Liu, X.-L. Lu and C.-R. Wu, J. Appl. Polym. Sci., 2013, 129, 1417-1425.

40 C. Marega and A. Marigo, Eur. Polym. J., 2003, 39, 1713-1720.

41 O. Hiroji and K. Keiko, Jpn. J. Appl. Phys., 1982, 21, L455.

42 K. Tashiro and M. Kobayashi, Phase Transitions, 1989, 18, 213-246.

43 J. P. Penning and R. St. John Manley, Macromolecules, 1996, 29, 84-90.

44 L. Yang, J. Ho, E. Allahyarov, R. Mu and L. Zhu, ACS Appl. Mater. Interfaces, 2015, 7, 19894-19905.

45 H. Yan, H. Zhang, R. Ubic, M. J. Reece, J. Liu, Z. Shen and Z. Zhang, Adv. Mater., 2005, 17, 1261-1265.

46 V. Cauda, S. Stassi, K. Bejtka and G. Canavese, ACS Appl. Mater. Interfaces, 2013, 5, 6430-6437. 